\title{
Analysis of the cis-acting requirements for germ-line-specific splicing of the P-element ORF2-ORF3 intron
}

\author{
Frank A. Laski ${ }^{1,3}$ and Gerald M. Rubin ${ }^{1,2}$ \\ ${ }^{1}$ Department of Biochemistry and ${ }^{2}$ Howard Hughes Medical Institute, University of California, Berkeley, California 94720 USA
}

P-element transposition is limited to the germ line because the element's third intron is only spliced in germ line cells. We show that a 240 -bp fragment containing this 190 -bp intron can confer germ line specificity when placed in the context of another gene. We find that the cis-acting regulatory sequences required for germ line regulation map near to, but not at, the $5^{\prime}$ or $3^{\prime}$ splice junctions.

[Key Words: Transposable element; P element; RNA splicing; intron]

Received January 24, 1989; revised version accepted March 4, 1989.

P elements are a family of transposable elements found in Drosophila melanogaster. They have been shown to be the causal agents of $\mathrm{P}-\mathrm{M}$ hybrid dysgenesis, a syndrome whose traits include high rates of sterility, mutation, and chromosomal rearrangements (Kidwell et al. 1977; for review, see Engels 1983). Autonomous P elements encode transposase, a trans-acting function that is necessary for P-element transposition and excision (Spradling and Rubin 1982; Engels 1984). P elements have been analyzed at the molecular level and found to range in size from $<500 \mathrm{bp}$ to the $2.9-\mathrm{kb}$ autonomous $P$ element (O'Hare and Rubin 1983). A typical P strain carries 30-50 P elements, of which approximately onethird are $2.9 \mathrm{~kb}$. The short nonautonomous $\mathrm{P}$ elements do not encode transposase activity but can be mobilized in trans when a source of transposase is supplied. P-element-mediated germ line transformation (Spradling and Rubin 1982; Rubin and Spradling 1982) allows one to introduce a mutated $\mathrm{P}$ element back into flies where the mutation's effect on P-element functions can be assessed. Using this strategy, Karess and Rubin (1984) have shown that all four open reading frames (ORFs) of the $P$ element are part of one cistron that encodes transposase.

P-element transposition is limited to the germ line. We have shown that this germ line specificity is achieved by a germ-line-specific splice that is required to join ORF2 with ORF3 (Fig. 1; Laski et al. 1986; Rio et al. 1986). Mutations that alter the consensus $5^{\prime}$ or $3^{\prime}$ splice sites of this intron abolish transposase production. Moreover, a mutation that precisely deletes the intron produces transposase in somatic tissues as well as the germ line. Thus, we were able to conclude that the inability of somatic cells to splice the intron that joins

${ }^{3}$ Present address: Department of Biology and Molecular Biology Institute, University of California, Los Angeles, California 90024 USA.
ORF2 to ORF3 is the sole basis for the germ line restriction of P-element transposition (see Fig. 1).

Although knowledge about the biochemistry of splicing is progressing rapidly (for review, see Sharp 1987; Green 1986; Padgett et al. 1986) and a number of alternative splices have been identified (for review, see Breitbart et al. 1987), little is known about the mecha$\mathrm{nism}(\mathrm{s})$ involved in the regulation of an alternative splice. Why are some introns spliced in all tissues at all times while others show specificity? What cis-acting sequences in the pre-mRNA are required for this specificity?

A parasitic element is under strong selective pressure to evolve a mechanism to limit its transposition to the germ line so that its spread through a population would not be impeded by needlessly lowering the viability of its hosts. Although we know that P elements have accomplished this objective by evolving a germ-line-specific splice, we do not understand how the tissue specificity of this splice is achieved. Three classes of models can be envisioned. First, it may be that the ORF2-ORF3 intron is not spliced in somatic cells simply because the splice recognition sequences are hidden by the tertiary structure adopted by the P-element transcript. In this model, a germ line factor, which itself may play no role in the splicing of host transcripts, is postulated to unfold the transcript and expose the intron. In the second class of models, a somatic factor specifically inhibits the ORF2-ORF3 splice. According to the third model, the normal splicing machinery is incapable of splicing the intron and requires additional positive acting factor(s) present only in the germ line.

In this paper we show that the 190-bp ORF2-ORF3 intron retains its germ line specificity when placed, as part of a 240-bp P-element fragment, into the context of a different gene. We also report the results of our initial 


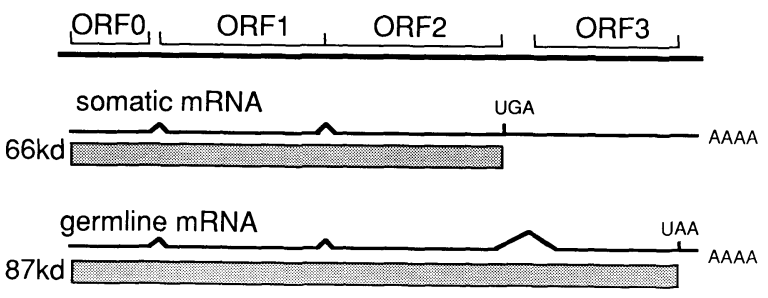

Figure 1. Structure of P-element mRNA and proteins. There are two major P-element RNA transcripts, the $2.5-\mathrm{kb}$ message made in somatic cells and a germ-line-specific message that contains a third splice that connects ORF2 to ORF3 in frame. The $2.5-\mathrm{kb}$ somatic message encodes a $66-\mathrm{kD}$ polypeptide, whereas the germ-line-specific message encodes the $87-\mathrm{kD}$ transposase protein (Laski et al. 1986; Rio et al. 1986).

mapping of the cis-acting sequences in the pre-mRNA that regulate the tissue specificity of splicing. We find that these sequences appear to map near, but not at, the splice junctions. These results argue against the splice being regulated by the tertiary structure of the message.

\section{Results}

There must be cis-acting sequences in the P-element pre-mRNA that allow the splicing machinery to distinguish between the ORF2-ORF3 intron and the other two P-element introns. Our experiments are designed to identify these sequences to elucidate their mode of action.

\section{Most of the ORF2-ORF3 intron sequences are not required for its germ-line-specific splicing}

To map whether or not the cis-acting regulatory sequences reside within the third (ORF2-ORF3) intron of the $\mathrm{P}$ element, the deletions diagramed in Figure 2 were constructed in Pc[ry]. Pc[ry] contains an active $P$ element marked with rosy (ry), an eye-color gene that encodes the enzyme xanthine dehydrogenase. It has been shown that on transformation Pc[ry] acts like a wildtype P element in Drosophila, producing high levels of transposase activity in the germ line but none in the soma (Karess and Rubin 1984). Using Pc[ry] we demonstrated previously that mutations destroying either the $5^{\prime}$ or $3^{\prime}$ splice junctions of the ORF2-ORF3 intron destroy the ability of the $P$ element to produce transposase and that a precise deletion of the ORF2-ORF3 intron creates a $\mathrm{P}$ element, $\mathrm{P}[\mathrm{ry}(\Delta 2-3)]$, that produces transposase in both the germ line and soma (Laski et al. 1986). We now use the same assays to determine which intron sequences are required in the pre-mRNA for the proper regulation of the germ-line-specific splice. If a $\mathrm{Pc}[\mathrm{ry}]$ construct containing one of the deletions retains germ line specificity for transposition, it will prove that the sequences deleted are not required in cis in the premRNA for the germ line specificity of the splice. However, if a deleted $\mathrm{P}$ element produces transposase in both the germ line and soma it would suggest that the deleted sequences are involved in the splicing specificity. A third possibility is that a deletion will destroy the intron, preventing it from being spliced in any tissue. Although this result provides data on what is required for a correct splice, it would not supply any direct information about what sequences are responsible for germ line specificity.

Transformant lines were assayed first for transposase activity in the germ line using the singed-weak $\left(s n^{w}\right)$ assay (see Methods). The $s n^{w}$ allele of the singed bristle locus results from the insertion of two nonautonomous P elements in inverted orientation (Roiha et al. 1988). In the absence of P-element transposase the $s n^{w}$ locus is very stable (all of the progeny are $s n^{w}$ ); however, when a source of transposase is provided in trans, $s n^{w}$ mutates at a high frequency to two new phenotypes (singed-plus, $s n^{+}$, and singed-extreme, sn ${ }^{e}$; Engels 1984). As seen in Figure 2, both lines carrying the control construct, wildtype Pc[ry], have high levels of germ line transposase activity $(46$ and $11 \%$ of the progeny of flies that carried both $\mathrm{Pc}[r y]$ and the $s n^{w}$ allele were either $s n^{e}$ or $s n^{+}$. The $\mathrm{P}[\mathrm{ry}(\Delta 2-3)]$ lines also have high levels of germ line activity. The presence of transposase activity in the soma was tested using the $\mathrm{P}[w]$ transformant $\mathrm{P}[\mathrm{w}(\mathrm{A})] 038$ (Laski et al. 1986; see Methods). $\mathrm{P}[w \mid \mathrm{A})] 038$ is homozygous for the white null allele $w^{1118}$ but has red eyes because it is transformed with a wild-type copy of the white locus. The wild-type white locus is carried within a nonautonomous P-element vector, and thus can excise only if transposase is provided in trans (Levis et al. 1985). Individual somatic excision events generate easily scored white patches in the red eye (Laski et al. 1986). The eyes of flies having one copy of $\mathrm{Pc}[\mathrm{ry}]$ and $\mathrm{P}[w]$ never show such patches (Laski et al. 1986; Fig. 2). However, flies having one copy of $\mathrm{P}[w]$ and $\mathrm{P}[\mathrm{ry}(\Delta 2-3)]$ have high rates of somatic excision. All of the eyes of this genotype examined (a total of 312 eyes from the three transformant lines) had a minimum of five patches per eye, although most of the eyes had many more excision events.

When the transposase assays were applied to flies carrying the deletion mutants, we found that three of the deleted P elements $(\Delta 1960-2030, \Delta 2000-2070$, and $\Delta 2027-2103$ ) are primarily germ line specific. All three were able to transpose stably into Drosophila without the benefit of a helper $\mathrm{P}$ element, and the resulting transformants had $s n^{w}$ destabilizing activity in the germ line (Fig. 2). Therefore, the ORF2-ORF3 intron of all three constructs must be able to be spliced in the germ line. Two of these deleted $P$ elements have wild-type levels of transposase activity, whereas a $P$ element carrying the $\Delta 2027-2103$ deletion appears to have reduced levels of transposase activity. The $\mathrm{P}[\mathrm{w}]$ assay shows that these three deleted $\mathrm{P}$ elements all have very low levels of transposase activity in the soma, as measured by their ability to induce somatic excision patches in the eyes of $\mathrm{P}[w \mid \mathrm{A})] 038$ flies (Fig. 2). Besides being infrequent, these patches were very small, many of them being only a few ommatidia in size. The soma/germ line index compares the amount of transposase activity present in the soma and germ line for each construct (Fig. 2). All three deleted constructs have a ratio of somatic to germ line ac- 




Figure 2. Mutations within the ORF2-ORF3 intron. At the top of the figure is the structure of Pc[ry]. The heavy line represents P-element sequences. The 7.2-kb HindIII rosy fragment is represented by the thin line. The structure of Pc[ry] is described in detail in Karess and Rubin (1984). An enlarged diagram of the region of P element that spans the ORF2-ORF3 intron is shown for Pc[ry] and other constructs. The locations of the $5^{\prime}$ and $3^{\prime}$ splice sites are designated by the arrows. The $\Delta 2-3$ deletion precisely removes the 190 -bp intron. The five constructs shown below $\mathrm{P}[\mathrm{ry} ; \Delta 2-3]$ contain deletions within the intron, the size and location of which are shown. For a precise description of these mutations see Methods. The $5^{\prime}$ consensus mutation mutates the T at 1951 and the A at 1953 to an A and T, respectively. The $3^{\prime}$ consensus mutation mutates the polypyrimidine tract as shown. For all constructs, multiple lines were assayed for transposase activity in both the germ line and soma. For the germ line assay, the number of $s n^{w}$ excision events divided by the total number of flies assayed is given. For the somatic assay, the total number of somatic excision patches divided by the total number of eyes assayed is given. The soma/germ line index displays the relative ratios of somatic transposase activity to germ line transposase activity. See Methods for an explanation of how this index was calculated. Our interpretation for each construct is shown also.

tivity $<3 \%$ that of the $\Delta 2-3$ construct. Therefore, the ORF2-ORF3 intron of these constructs is regulated in a germ-line-specific manner, although the deletions have weakened this regulation. Because the splicing of the introns is still being regulated, the cis-acting sequences required for this regulation must map outside of the deleted regions. We believe that the deletions produce a 'leakiness' in the regulation, perhaps as an effect of the reduction in the size of the intron. It is also possible that cis-acting sequences mapping within the deleted region play a minor role in the regulation; however, the most important sequences must map outside of this region.

Two of the deleted P elements lack measurable transposase activity in both the germ line and soma. One of these deletions ( $\Delta 1960-2103)$ combines two of the above deletions $(\Delta 1960-2030$ and $\Delta 2027-2103)$ making the intron only 47 bases long. Although other Drosophila introns are this small, it appears that the size reduction disables the ORF2-ORF3 intron. The $\Delta 2027-$ 2117 construct also appears unable to be spliced in either the germ line or soma. There is no data on where the branchpoint of the ORF2-ORF3 intron is located, but a branchpoint consensus sequence (TTAAT) is found at positions 2111 to 2115 . It is possible that the $\Delta 2027$ 2117 construct is not spliced because the branchpoint has been removed.

\section{Germ line specificity is not encoded in the $5^{\prime}$ and $3^{\prime}$ splice site sequences}

Although the $5^{\prime}$ and $3^{\prime}$ splice sites do conform to the consensus splice site sequences, it is possible that their 
precise sequence plays a role in the germ line specificity. To test this hypothesis both splice sites were independently mutated, and the effect on germ line specificity tested. The $5^{\prime}$ splice site has 6 -bp out of 9-bp identity with the consensus sequence proposed by Mount and Steitz (1984; see Fig. 2). We mutated the $5^{\prime}$ splice site toward the consensus, reasoning that a splice site of perfect consensus would be unlikely to be a cis-acting signal for germ line specificity. The altered splice site has 8 bp of the 9 -bp consensus sequence (Fig. 2). To have all 9 bp meet consensus would require a change at position 1945 from a $\mathrm{T}$ to a $\mathrm{C}$ or an $\mathrm{A}$. Either mutation results in an amino acid change in ORF2, and might therefore inactivate the $87-\mathrm{kD}$ transposase. The $3^{\prime}$ splice site has a 12-bp out of 15-bp homology with the consensus sequence (Mount and Steitz 1984). We mutated all 11 bases in the polypyrimidine tract (see Fig. 2). As shown in Figure 2, the $\mathrm{P}\left[\mathrm{ry},\left(5^{\prime}\right.\right.$ consensus $\left.)\right]$ and $\mathrm{P}\left[\mathrm{ry},\left(3^{\prime}\right.\right.$ consensus)] transformants have high levels of transposase activity in the germ line but only a minimal amount in the soma. Therefore, we believe that the $5^{\prime}$ and $3^{\prime}$ splice site sequences of the ORF2-ORF3 intron are not involved directly in the germ line specificity of the splice.

\section{$240 \mathrm{bp}$ is sufficient to confer germ-line-specific splicing}

Exon sequences are known to be able to affect the regulation of alternative splicing (e.g., see Reed and Maniatis 1986). It is therefore possible that the cis-acting regula- tory sequences are located outside of the intron in the adjacent protein-coding sequences. The assay we used to examine the intron mutations is not suitable for testing mutations in the exon sequences, however, because any changes in the ORF2 or ORF3 coding sequences are likely to inactivate transposase activity and therefore the assay. Instead, we devised a histochemical assay to measure the germ-line-specific splice. P-element sequences containing the ORF2-ORF3 intron were cloned in between the lacZ gene, which encodes $\beta$-galactosidase ( $\beta$-gal), and the hsp70 promoter and about $1 \mathrm{~kb}$ of hsp70 protein-coding sequences (Fig. 3). The hsp70 coding sequences are joined in frame to ORF2 at the PstI site at nucleotide 1911 in $\mathrm{P}$ element. The lacZ gene is cloned in frame to ORF3 at a BamHI site that was inserted into the P-element sequences at nucleotide 2183. For $1 a c Z$ to be expressed from this construct, the hsp70 promoter must be activated and the ORF2-ORF3 intron must be spliced out. Therefore, in a heat-shocked fly carrying this construct, the presence of $\beta$-gal activity will identify tissues in which the ORF2-ORF3 intron can be spliced. This gene construct, called hsp70$\mathrm{P}(1911-2183)-1 a c Z$, was cloned into a P-element vector and transformed into flies. Transformants were also generated with the control construct hsp70-P(1911$(\Delta 2-3)-2183)-1 a c Z$, which differs from hsp70-P(19112183 )-lacZ only in that it contains the $\Delta 2-3$ deletion and therefore should express $\beta$-gal in all tissues where the $h s p 70$ promoter is active.

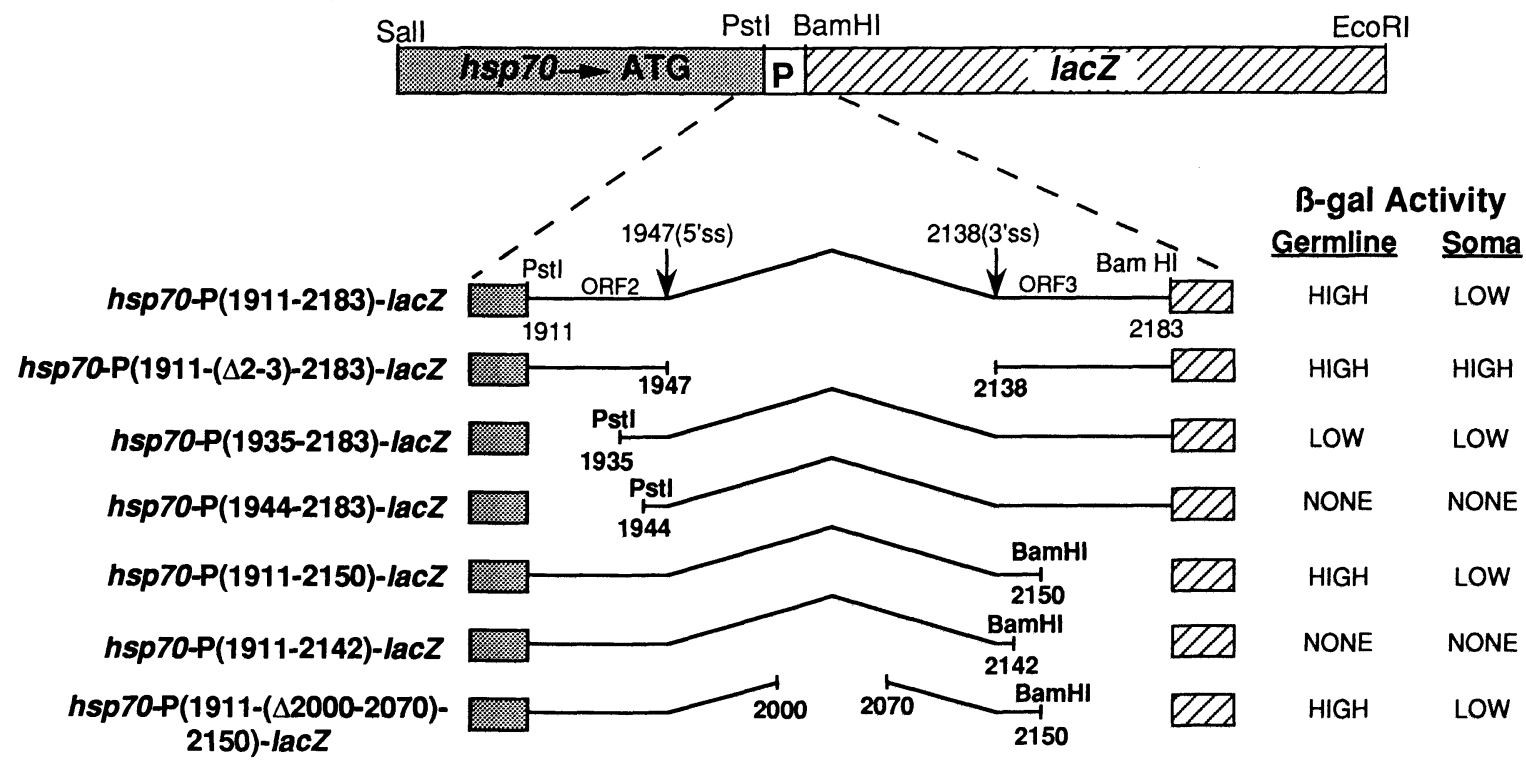

Figure 3. Constructs used for histochemical assays of the germ-line-specificity of the ORF2-ORF3 splice. At the top of the figure the structure of the gene construct $h s p 70-\mathrm{P}(1911-2183)-l a c Z$ is diagramed; for transformation into flies the gene construct was inserted into the P-element transformation vector, pDM30 (see Methods). This construct consists of the following three fragments of DNA. First, a 2.5-kb SalI-PstI fragment from pHT1 (Pelham 1982), which contains the hsp 70 promoter, the translation initiation codon ATG, and 940 bp of heat-shock coding sequences. The Sall site is from the plasmid vector of pHT1 and the PstI site is located at nucleotide 1190 of the hsp 70 sequence (Karch et al. 1981). Second, a 273-bp PstI-BamHI fragment from the P element; the PstI site is at nucleotide 1911 and the BamHI site is at position 2183 (see Methods). Finally, a 4.25-kb BamHI-EcoRI fragment from pC4-Bgal (Thummel et al. 1988), which contains the E. coli lacZ gene and a region of SV40 containing an intron and a polyadenylation site. A blowup of the P-element sequences from this construct and others are shown. For each construct the relative amounts of $\beta$-gal activity in the germ line and soma is summarized. See Fig. 4 and text for details. 


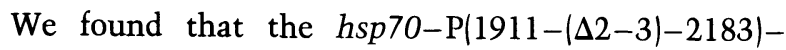
lacZ construct expressed $\beta$-gal at high levels in all tissues (Fig. 4A,C,E,H) whereas the hsp70-P(19112183 - - lac $Z$ construct expressed $\beta$-gal preferentially in the germ line. High-level expression of $\beta$-gal from hsp70-P(1911-2183)-lacZ was seen only in the ovaries and at the tip of the testes, although there was a low level of somatic expression (Fig. 4B,D,F,I). This result maps the cis-acting P-element sequences sufficient for germ-line-specific splicing to between nucleotides 1911 and 2183.

Using the $\beta$-gal expression assay we continued to map the intron sequences using the constructs shown in Figure 3. Construct hsp70-P(1911-2150)-lacZ is identical to $h s p 70-\mathrm{P}(1911-2183)-l a c Z$, except that it does not contain the P-element exon sequences from 2151 to 2183 (Fig. 3). This construct has an identical germ-linespecific staining pattern as $h s p 70-\mathrm{P}(1911-2183)-1 a c Z$ (data not shown); therefore the deletion from 2150 to 2183 did not remove any sequences necessary for the germ line specificity. Further deletion of $69 \mathrm{bp}$ from the center of the 190-bp intron (construct hsp70-P(1911(42000-2070)-2150)-lacZ; Fig. 3) also did not affect germ line specificity (data not shown). This contrasts with the results we obtained with constructs $h s p 70$ $\mathrm{P}(1911-2142)-l a c Z$ and hsp70-P(1944-2183)-lacZ (Fig. 3), which reduce the size of the P-element exon sequences present to $5 \mathrm{bp} 3^{\prime}$ or $4 \mathrm{bp} 5^{\prime}$ of the intron, respectively. After heat shock, we could detect no $\beta$-gal activity in any tissue in adult flies containing these constructs (data not shown), suggesting that the ORF2-ORF3 intron had been inactivated by the mutations. We assume that this is a direct result of deleting sequences important to the splice sites or of bringing inhibitory sequences too close to these sites.

Transformed flies containing the construct hsp70$\mathrm{P}(1935-2183)-$ lac $Z$ have a novel phenotype. They express a low level of $\beta$-gal activity in all tissues; the pattern is clearly not germ line specific. Ovaries from flies carrying this construct stain at a much reduced level

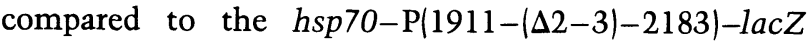
construct and have limited staining of the early staged egg chambers (Fig. 4J). The very low level of somatic staining is similar to that generated by the hsp70-
$\mathrm{P}(1911-2183)-1 a c Z$ construct but the higher level of staining in the ovaries is absent (cf. panels I and J, Fig. 4).

P-element transposition is repressed in $\mathrm{P}$ strains (Engels 1983). Given that transposase production requires that the ORF2-ORF3 splice be made, regulation of this splice would be an obvious point to exert repression. Therefore, we examined whether the level of $\beta$-gal expression from $h s p 70-\mathrm{P}(1911-2183)-1 a c Z$ was affected when this construct was placed in a $P$ strain $(\pi 2)$ environment where transposition is known to be repressed. Contrary to a model where the $\mathrm{P}$ cytotype strongly suppresses the ORF2-ORF3 splice, $\beta$-gal synthesis still occurred, although at a slightly reduced level (data not shown). A partial suppression of the splice, therefore, has not been ruled out.

\section{Discussion}

P-element transposition occurs at high levels in the germ line, but not in somatic tissue. Previously we demonstrated that this tissue specificity is controlled at the level of mRNA splicing (Laski et al. 1986). In this paper we present data mapping the cis-acting sequences that are required for the germ line specificity of the splice. In Figure 5 are the P-element sequences present in the hsp70-P(1911-2183)-lacZ gene construct. The data from this paper show that a 240-bp fragment consisting of the P-element sequences from 1911 to 2150, which includes the 190-bp ORF2-ORF3 intron, is able to confer germ line specifity in the context of another gene. Therefore, we believe the sequences required in cis for the germ line specificity of the splice must be located within this 240 -bp fragment. We also show that $\mathrm{P}$ elements containing deletions covering most of the ORF2ORF3 intron (between 1960 and 2103) still retain their germ line specificity. We have also shown that although the $5^{\prime}$ and $3^{\prime}$ splice junctions are intimately involved in the ORF2-ORF3 splice, they do not appear to have a direct role in regulating germ line specificity. Taken together, these data map the cis-acting regulatory sequences to within the 73 bases highlighted in Figure 5. We realize, however, that there is a problem with combining the data from several constructs in this way. If

Figure 4. $\beta$-Galactosidase assay for germ-line-specific splicing. Heat-shocked flies were stained in a solution containing X-Gal for 2 $\mathrm{hr}$ and photographed. Shown are the stained body $(A, B)$, ovaries $(C, D)$, and testes $(E, F)$ from flies transformed with $h s p 70-\mathrm{P}(1911-$ $(\Delta 2-3)-2183)-1 a c Z(A-C)$ or hsp 70-P(1911-2183)-lacZ $(D-F)$. The arrows in $F$ point to the lac $Z$ staining at the tips of the testes. Also shown are stained ovaries from ry 506 flies $(G)$ and flies transformed with the $h s p 70-\mathrm{P}(1911-2183)-1 a c Z(H)$, hsp $70-\mathrm{P}(1911-$ $(\Delta 2-3)-2183)-1 a c Z(I)$, and hsp70-P(1935-2183)-lacZ $(J)$ constructs. A comparison of $A$ and $B$ shows a much higher level of $\beta$-gal expression in the dissected body (somatic tissue) of the construct containing the $\Delta 2-3$ deletion than that without the deletion. The ovaries from the $h s p 70-\mathrm{P}(1911-(\Delta 2-3)-2183)-1 a c Z$ transformant stain intensely with X-Gal $(C, H)$. Much of this staining is in the follicle cells which are on the surface of the ovaries and are somatic. These follicle cells stain at a much reduced level in the hsp 70-P(1911-2183)-lacZ transformants, leaving only the early staged egg chambers of the ovary (which are germ line) staining darkly $(D, I)$. Within the testes it has been shown previously that the $h s p 70$ promoter is active only at the tips of the testes (Bonner et al. 1984). We confirmed this result with the $h s p 70-\mathrm{P}(1911-(\Delta 2-3)-2183)-1 a c Z$ construct which expresses $\beta$-gal primarily at the tips of the testes $(E)$. The other darkly staining tissues are the seminal vesicles which are somatic. The hsp $70-\mathrm{P}(1911-2183)-l a c Z$ strain also expresses $\beta$-gal at the tips of the testes, but the seminal vesicles do not stain. We conclude from this data that the hsp $70-$ $\mathrm{P}(1911-2183)-1 a c Z$ gene construct is being expressed in a germ-line-specific manner, although there is a low level of somatic expression. 

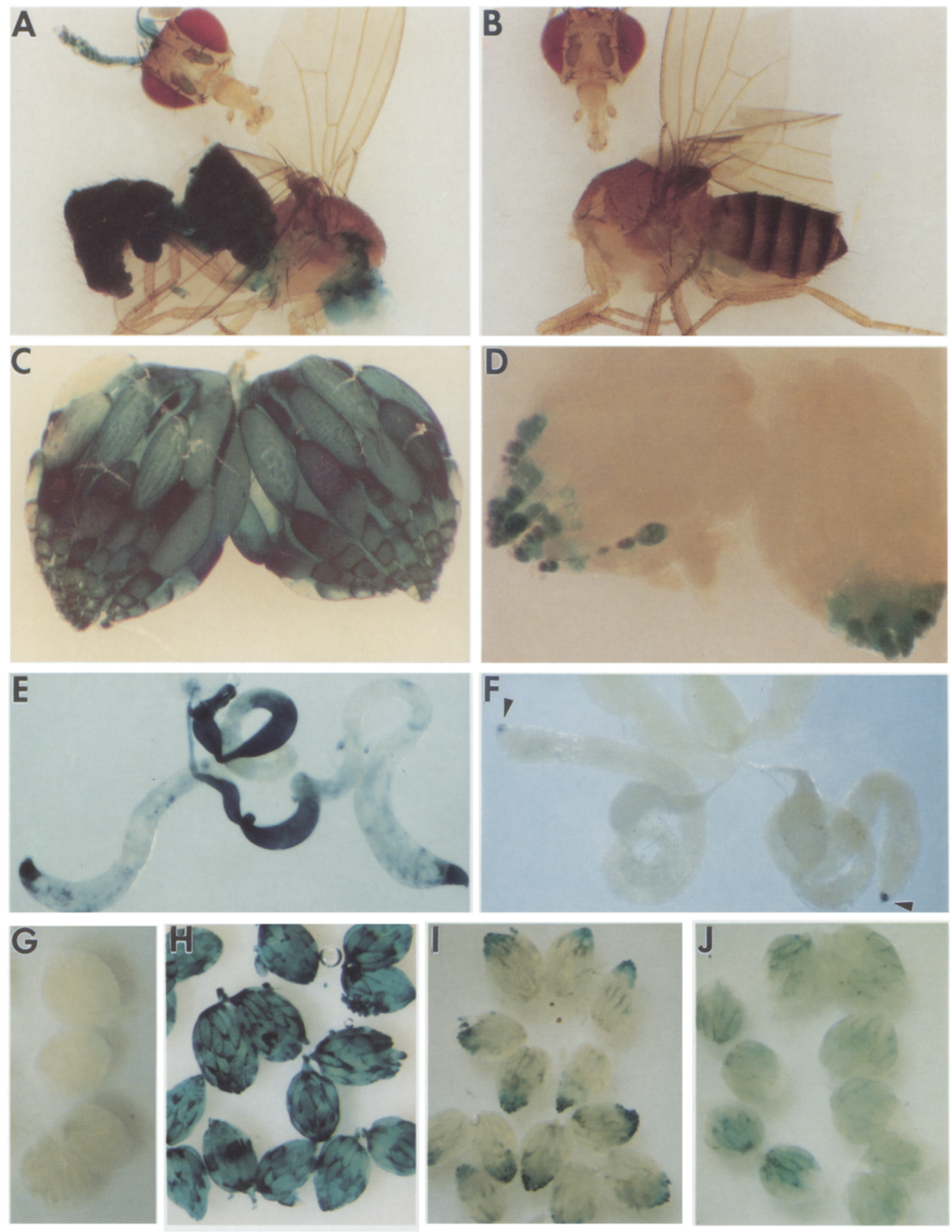

Figure 4. (See facing page for legend.) 
two redundant sequences are independently capable of regulating the germ line specificity of the splice, then these sequences might escape detection if only one of them is mutated at a time.

The germ line specificity of P-element transposition is controlled tightly (Engels 1983; McElwain 1986). Many of the constructs tested retain germ line specificity, but the tightness of the somatic inhibition is reduced. The cause of this leakiness is unknown and may be different for the different constructs. Three of the Pc[ry] constructs carrying deletions within the intron show low levels of transposase in the soma. Because the leakiness is very slight and because two of the deletions $(\Delta 1960$ 2030 and $\Delta 2027-2103$ ) overlap by only two bases, we do not believe that the leakiness is caused by the deletion of any cis-acting regulatory sequences that are involved directly in the regulation of the splice. Perhaps the leakiness is caused by a reduction in the size of the intron, which could have an effect on the optimal regulation of the splice. The 5' consensus mutation also resulted in a low level of somatic leakiness of transposase activity. There are many possible explanations for this. As an example, it is possible that mutating the $5^{\prime}$ splice site toward the consensus increases the relative strength of the splice and allows it to overcome slightly an inhibiting factor present in the soma. The lac $Z$ constructs have a significant amount of leakiness in the soma, estimated at $5-10 \%$ of the expression seen in the lac $Z$ construct containing the $\Delta 2-3$ deletion. It is possible that this leakiness is not caused by a low level of somatic splicing but rather by a low level of internal initiation of translation within the lacZ gene. We think this is unlikely because two other lacZ transformants [hsp 70-P/19442183)-lacZ and hsp 70-P(1911-2142)-lacZ] make very similar transcripts but do not express $\beta$-gal. Rather, we think that either the overall structure of the hybrid message interferes with the proper regulation of the splicing machinery, or a minor part of the cis-acting regulatory sequences is located outside of nucleotides 1911 to 2183 .

One of our hopes was that the identification of the cis-acting regulatory sequences would suggest a mechanism for the regulation of the germ-line-specific splice. Although we have not yet fully defined the cis-acting sequences, we believe that our results argue for some models and against others. It has been shown that the tertiary structure of a transcript can affect its alternative splicing (Solnick 1985). One model for the germ-linespecific regulation of the P-element splice is that the tertiary structure of P-element RNA in the germ line is different from that in the somatic tissue. The P-element RNA transcript might normally be folded into a structure that prevents access of the normal splicing apparatus to the ORF2-ORF3 splice. In germ line tissue the structure of the RNA might be altered (e.g., by the presence of an RNA binding protein), allowing the normal splicing machinery to act. We believe our data argue strongly against this model and any model that involves large secondary or tertiary structures of the pre-mRNA. If the tertiary structure of the pre-mRNA is crucial then the deletions within the intron in the Pc[ry] constructs or the deletions of most of the P-element sequences in the $1 a c Z$ constructs would have been expected to destroy the structure and thus abolish the regulation. Therefore, we believe our data favors models in which a factor(s) interacts with the P-element pre-mRNA at a specific and local sequence(s). This factor could either be germline-specific and be required for the splice or it could be limited to the soma and inhibit the splice. An obvious candidate is the small nuclear ribonucleoproteins (snRNPs), many of which are involved intimately in mRNA splicing (for review, see Maniatis and Reed 1987). It is thought that some of these snRNPs function by interacting with the $5^{\prime}$ splice site, the $3^{\prime}$ splice site, and the branchpoint. However, our data argue that the $5^{\prime}$ and $3^{\prime}$ splice sites are not involved in the germ line specificity of the splice. We have no data on whether the branchpoint is involved in the regulation of the germ line specificity of the splice. In yeast the branchpoint has a fixed sequence, TACTAAC (Rodriguez et al. 1984). In higher eukaryotes, including Drosophila, the consensus sequence for branchpoints is much weaker. In Drosophila the consensus sequence is $\mathrm{C}^{\mathrm{T}}-\mathrm{T}_{-} /{ }_{\mathrm{A}}{ }^{\mathrm{G}}-\mathrm{A}-{ }_{\mathrm{C}} /{ }^{\mathrm{T}}$ (Keller and Noon 1985). A sequence fitting this consensus is the TTAAT located at nucleotide 2111 to 2115; however, there is no biochemical data on whether this is the branchpoint. When these sequences are removed in the $\mathrm{P}[\mathrm{ry}(\Delta 2027-2117)]$ construct, no transposase activity is observed in any tissue. This is probably a result of the inactivation of the intron, but it is unclear whether this inactivation results from deleting the branchpoint.

A potential site for the cis-acting regulatory region is the sequences upstream from the $5^{\prime}$ splice junction. Deletion of nucleotides 1911-2134 gave a unique result. The hsp 70-P(1935-2183)-lacZ construct has low levels of $\beta$-gal activity in the soma, an amount equivalent to the leakiness seen in the $h s p 70-\mathrm{P}(1911-2183)-$ ? acZ construct, but has little expression in the germ line. The fact that there still is the somatic leakiness suggests that deleting the sequences $5^{\prime}$ to 1935 did not inactivate the intron. Rather it appears that the deletion preferentially inhibits the intron from being spliced in the germ line either by removing a required cis-acting regulatory sequences or by bringing inhibitory sequences too close to the intron.

\section{Methods}

Drosophila strains

All Drosophila strains used are described in Laski et al. (1986).

\section{Plasmid construction}

All P-element nucleotide positions listed are in reference to the published sequence of the $2.9-\mathrm{kb}$ autonomous $\mathrm{P}$ element (O'Hare and Rubin 1983).

M13-R1-Sal was constructed by cloning the 669-bp EcoRISalI fragment from Pc[ry] (Karess and Rubin 1984) into EcoRISall digested M13mp18 (Yanisch-Perron et al. 1985). Singlestranded DNA was isolated and site-specific mutagenesis was 

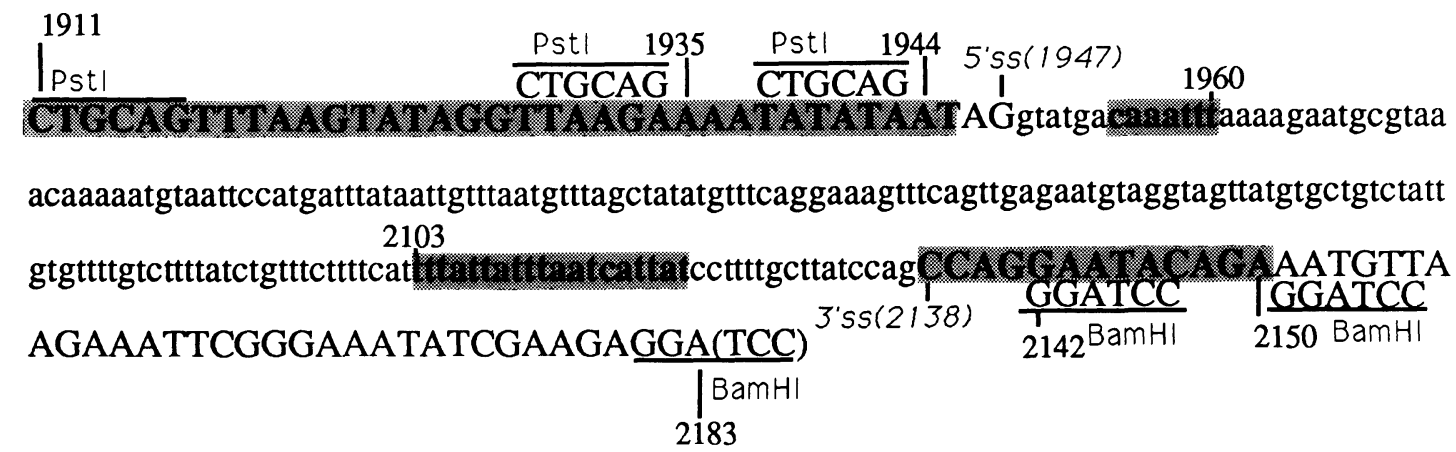

Figure 5. DNA sequence of the 273-bp region of the $\mathrm{P}$ element that confers germ line specificity to the $h s p 70-\mathrm{P}(1911-2183)-1 a c Z$ gene construct. Capital letters are exon sequences; lowercase letters are intron sequences. The location of the $5^{\prime}$ and $3^{\prime}$ splice sites are shown. The location of the PstI sites and BamHI sites introduced by in vitro mutagenesis (see Methods) are shown. The cis-acting regulatory sequences have been mapped to within the 73 highlighted bases (see text).

carried out using a minor modification of the procedures described by Hutchison et al. (1978), Razin et al. (1978), and Kudo et al. (1981). Oligonucleotides used for mutagenesis were: 5'-AAGACAAAACACAATTATAAATCATG for $\triangle 2000-2070$; 5'-CTGAAACTTTCTAGAAACATATAG for the $X b a \mathrm{I}$ site at 2030; 5'-ATGATTAAATAATAAAAATTTGTCATACCT for 1960-2103, 5'-TTTTAAATTTGACTTACCTATTAT for the $5^{\prime}$ consensus mutation, and $5^{\prime}$-TCTGTATTCCTGGCTGAGAGGAAGGGGAGATAATGATTAAATAA for the $3^{\prime}$ consensus mutation. These mutations were cloned back into Pc[ry] as previousely described (Laski et al. 1986). These plasmids are

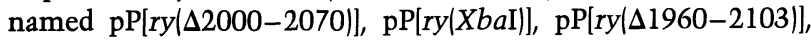
$\mathrm{pP}\left[\mathrm{ry} \mid 5^{\prime}\right.$ consensus $\left.)\right]$, and $\mathrm{pP}\left[\mathrm{ry}\left(3^{\prime}\right.\right.$ consensus $\left.)\right]$, respectively.

$\mathrm{P}[\mathrm{ry}(\mathrm{XbaI})]$ is identical to $\mathrm{Pc}[\mathrm{ry}]$ except for the unique $\mathrm{XbaI}$ site at 2030. $\mathrm{P}[r y(X b a \mathrm{I})]$ was linearized with $\mathrm{XbaI}$ and digested with exonuclease Bal31. XbaI linkers (CTCTAGAG) were ligated to the DNA, which was digested with $X b a \mathrm{I}$, followed by circularization with ligase and transformation into $E$. coli. The extent of the Bal31 deletion in individual colonies was analyzed, first by agarose gel and then by DNA sequence analysis. Three deletions were selected for subcloning back into Pc[ry]. One of these deletions put the $\mathrm{XbaI}$ linker to the $3^{\prime}$ side of nucleotide 1960. This construct was digested with EcoRI and $X b a I$, and the 249-bp fragment (from the EcoRI site at 1712 to the $X b a I$ site at 1960 ) was subcloned between the EcoRI site at 1712 and the $X b a I$ site at 2030 of $\mathrm{P}[\mathrm{ry}(\mathrm{XbaI})]$. The resulting construct $\mathrm{pP}[r y(\Delta 1960-2030)]$ is identical to Pc[ry], except nucleotides 1961 through 2029 are deleted and additional linker sequences are inserted. The sequence across the deletion junction is (1960)TctctaGA(2031) where the uppercase letters are P-element sequences, the lowercase letters are additional sequences resulting from the cloning steps, and the $X b a I$ site is underlined. Two other deletions put the $X b a I$ site to the $5^{\prime}$ side of nucleotide 2103 and 2117 . The $X b a I-S a l$ fragment from these mutants were subcloned between the $X b a I$ site at 2030 and the Sall site at 2411 of $\mathrm{P}[\mathrm{ry}(\mathrm{XbaI})]$. The resulting constructs are called pP[ry|(2027-2103)] and $\mathrm{pP}[r y(\Delta 2027-2117)]$. The sequence across the deletion junction of $\Delta 2027-\mathrm{XbaI}-2103$ is (2026)TCtagagcctcgacgT(2103). The additional nucleotides were added during the construction in an unplanned and unknown way. The sequence across the deletion junction of $\Delta 2027-X b a I-2117$ is (2026)TCtagagA(2117).

Single-stranded M13-RI-Sal was mutagenized with the following oligonucleotides: 5'-GCTTTCAGAGGGATCCTCTTCGAT for the BamHI site at 2183; 5'-CGAATTTCTTGGATCCTCTGTATTCC for the BamHI site at $2150 ; 5^{\prime}$ TCTTAACATTTCTGGATCCCTGGC for the BamHI site at
2142. The M13-RI-Sal clone containing the BamHI site at 2183 was further mutagenized with the following oligonucleotides: 5'-TTATATATTTCTGCAGCCTATACTTA for the Pst site at 1935 and 5'-GTCATACCTATCTGCAGTTTTCTTAAC for the PstI site at 1944. The M13-RI-Sal clone containing the $\mathrm{BamHI}$ site at 2150 was further mutagenized, inserting the 12000-2070 mutation. The PstI-BamHI fragment from all of these constructs was subcloned into the hsp70-P(1911-2183)lac $Z$ construct (see Fig. 3). The hsp70-P-lacZ constructs were subcloned between the SalI and EcoRI site in the polylinker of pHSX (K. Jones and G. Rubin, unpubl.) which is a modified form of pHSS7 (Seifert et al. 1986). These constructs were digested with NotI (which flank the polylinker of pHSX) and the fragments cloned into the NotI site of pDM30 (Mismer and Rubin 1987).

\section{P-element transformation}

P-element transformation was as in Karess and Rubin (1984).

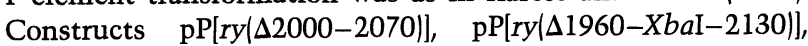
$\mathrm{pP}[r y(\Delta 2027-X b a I-2103)], \mathrm{pP}\left[r y\left(5^{\prime}\right.\right.$ consensus $\left.)\right], \mathrm{pP}\left[r y\left(3^{\prime}\right.\right.$ consensus)] were injected without a helper $P$ element. The other constructs were transformed using $\mathrm{p} \pi 25.7 \mathrm{wc}$ (Karess and Rubin 1984).

\section{Transposase assays}

Germ line and somatic transposase assays were described previously (Laski et al. 1986). For the germ line assay a male $\left(G_{0}\right)$ carrying the $\mathrm{P}[r y]$ construct to be assayed is crossed to y $s n^{w} ; r y^{506}$ females. Three to eight individual male progeny $\left(G_{1}\right)$ were crossed independently to $y s n^{3} v$ females. The progeny of this cross $\left(G_{2}\right)$ were assayed for $s n^{w}, s n^{+}$, and $s n^{e}$ bristles. For the somatic assay a male $\left(G_{0}\right)$ carrying the $P[r y]$ construct to be assayed was crossed to $\mathrm{P}[(w) \mathrm{A}] 038$ females, and the male progeny $\left(G_{1}\right)$ was assayed for mosaic eyes. The assay was quantified by counting the number of mosaic eyes and the number of mosaic patches per eye. Most mosaic patches are white; however, small mosaic patches appeared black. To be scored as a mosaic patch, there must be at least two black ommatidia contiguous to each other. Multiple mosaic patches per eye were scored whenever two patches were not adjoining. The soma/ germ line index was calculated by dividing the somatic excision rate (total number of mosaic spots divided by the total number of eyes examined) by the germ line excision rate (total number of $s n^{+}$and $s n^{e}$ flies divided by the total number of flies examined). The index was normalized to give $\mathrm{P}[r y ; \Delta 2-3]$ the defined 
rate of 1 (equal levels of transposase activity in the germ line and soma).

\section{ß-Gal staining}

Flies were heat-shocked at $37^{\circ} \mathrm{C}$ for $1 \mathrm{hr}$, followed by $90 \mathrm{~min}$ at $25^{\circ} \mathrm{C}$, followed by a second $37^{\circ} \mathrm{C}$ heat shock for $30 \mathrm{~min}$. The flies were dipped in $95 \%$ ethanol (to wet their wings), dissected in $0.7 \mathrm{M} \mathrm{NaCl}$, and stained at room temperature in a microtiter well. To make the staining solution, $27.5 \mathrm{ml}$ of $0.1 \mathrm{M}$ citric acid and $27.6 \mathrm{~g}$ of $\mathrm{Na}_{2} \mathrm{HPO}_{4}$ were diluted to 1 liter with $\mathrm{H}_{2} \mathrm{O}$. Two milliliters of this solution was mixed with $0.25 \mathrm{ml}$ of $50 \mathrm{mM}$ potassium ferricyanide, $0.25 \mathrm{ml}$ of $50 \mathrm{~mm}$ potassium ferrocyanide, and $25 \mu \mathrm{l}$ of a $10 \%$ solution of X-Gal (5-bromo,4chloro-3-indolyl- $\beta$-D-galactopyranoside) dissolved in dimethylformamide.

\section{Acknowedgments}

We thank Tom Lila for help with the transformation of the Pelement constructs. We are grateful to Carl Thummel for providing us with the pC4- $\beta$ gal construct and advice on its use. We thank members of the Rubin lab for their help and advise. We thank Don Rio and members of the Laski lab for critical reading of the manuscript. F.A.L. was an Exxon Education Foundation Fellow of the Life Sciences Research Foundation. This work was supported by grants from the Life Sciences Research Foundation and the National Institutes of Health (NIH) to F.A.L. and from the NIH to G.M.R.

\section{References}

Bonner, J.J., C. Parks, J. Parker-Thornburg, M.A. Mortin, and H.R.B. Pelham. 1984. The use of promoter fusions in Drosophila genetics: Isolation of mutations affecting heat shock response. Cell 37: 979-991.

Breitbart, R.E., A. Andreandis, B. Nadal-Ginard. 1987. Alternative splicing: A ubiquitous mechanism for the generation of multiple protein isoform from single genes. Annu. Rev. Biochem. 56: 467-495.

Engels, W.R. 1983. The P family of transposable elements in Drosophila. Annu. Rev. Genet. 17: 315-344.

- 1984. A trans-acting product needed for P factor transposition in Drosophila. Science 226: 1194-1196.

Green, M.R. 1986. Pre-mRNA splicing. Annu. Rev. Genet. 20: $671-708$.

Hutchison, C.A., S. Phillips, M.H. Edgell, S. Gillam, P. Jahnke, and M. Smith. 1978. Mutagenesis at a specific position in a DNA sequence. J. Biol. Chem. 253: 6551-6560.

Karch, F., I. Torok, and A. Tissieres. 1981. Extensive regions of homology in front of the two heat shock variant genes in Drosophila melanogaster. J. Mol. Biol. 148: 219-230.

Karess, R.E. and G.M. Rubin. 1984. Analysis of P transposable element functions in Drosophila. Cell 38: 135-146.

Keller, E.B. and W.A. Noon. 1985. Intron splicing: A conserved internal signal in introns of Drosophila melanogaster. $\mathrm{Nu}$ cleic Acids Res. 13: 4971-4981.

Kidwell, M.G., J.F. Kidwell, and J.A. Sved. 1977. Hybrid dysgenesis in Drosophila melanogaster: A syndrome of aberrant traits including mutation, sterility, and male recombination. Genetics 36: 813-833.

Kudo, I., M. Leineweber, and U.L. RajBhandary. 1981. Site-specific mutagenesis on cloned DNAs: Generation of a mutant of Escherichia coli tyrosine suppressor tRNA in which the sequence G-T-T-C corresponding to the universal G-T-II-C sequence of tRNAs is changed to G-A-T-C. Proc. Natl. Acad. Sci. 78: 4753-4757.
Laski, F.A., D.C. Rio, and G.M. Rubin. 1986. Tissue Specificity of Drosophila P element transposition is regulated at the level of mRNA splicing. Cell 44: 7-19.

Levis, R., T. Hazelrigg, and G.M. Rubin. 1985. Effects of genomic position on the expression of transduced copies of the white gene of Drosophila. Science 229: 558-560.

Maniatis, T. and R. Reed. 1987. The role of small nuclear ribonuclearprotein particles in pre-mRNA splicing. Nature 325: 673-678.

McElwain, M.C. 1986. The absence of somatic effects of P-M hybrid dysgenesis in Drosophila melanogaster. Genetics 113: 897-918.

Mismer, D. and G.M. Rubin. 1987. Analysis of the promoter of the nina E opsin gene in Drosophila melanogaster. Genetics 116: $565-578$.

Mount, S.M. and J.A. Steitz. 1984. RNA splicing and the involvement of small ribonucleoproteins. Modern Cell Biol. 3: 249-297.

O'Hare, K. and G.M. Rubin. 1983. Structures of P transposable elements and their sites of insertion and excision in the Drosophila melanogaster genome. Cell 34: 25-35.

Padgett, R.A., P.J. Grabowski, M.M. Konarska, S. Seiler, and P.A. Sharp. 1986 Splicing of messenger RNA precursors. Annu. Rev. Biochem. 55: 1119-1150.

Pelham, H. R. B. 1983. A regulatory upstream promoter element in the Drosophila hsp70 heat-shock gene. Cell 30: $517-528$.

Razin, A., T. Hirose, K. Itakura, and A.D. Riggs. 1978. Efficient correction of a mutation by use of chemically synthesized DNA. Proc. Nat1. Acad. Sci. 75: 4268-4270.

Reed, R. and T. Maniatis. 1986 A role for exon sequences and splice-site proximity in splice-site selection. Cell 46: 681690.

Rio, D.C., F.A. Laski, and G.M. Rubin. 1986. Identification and immunochemical analysis of biologically active Drosophila P element transposase. Cell 44: 21-32.

Rodriguez, J. R., C.W. Pikielny, and M. Rosbash. 1984. In vivo characterization of yeast mRNA processing intermediates. Cell 39: 603-610.

Roiha, H., G.M. Rubin, and K. O'Hare. 1988. P element insertions and rearrangements at the singed locus of Drosophila melanogaster. Genetics 119: 75-83.

Rubin, G.M. and A.C. Spradling. 1982. Genetic transformation of Drosophila with transposable element vectors. Science 218: $348-353$.

Seifert, H.S., E.Y. Chen, M. So, and F. Hefron. 1986. Shuttle mutagenesis: A method of transposon mutagenesis for Saccharomyces cerevisiae. Proc. Natl. Acad. Sci. 83: 735-739.

Sharp, P.A. 1987. Splicing of messenger RNA precursors. Science 235: 766-771.

Solnick, D. 1985. Alternative splicing caused by RNA secondary structures. Cell 43: 667-676.

Spradling, A.C. and G.M. Rubin. 1982. Transposition of cloned $P$ elements into Drosophila germ line chromosomes. Science 218: 341-347.

Thummel, C.S., A.M. Boulet, and H.D. Lipshitz. 1988. Vectors for Drosophila $P$ element-mediated transformation and tissue culture transfection. Gene 74: 445-456.

Yanisch-Perron, C., J. Vieira, and J. Messing. 1985. Improved M13 phage cloning vectors and host strains: Nucleotide sequences of the M13mpl8 and pUC19 vectors. Gene 33: 103-119. 


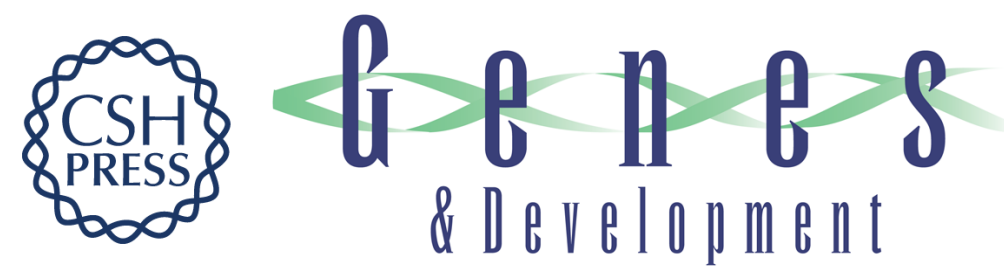

\section{Analysis of the cis-acting requirements for germ-line-specific splicing of the P-element ORF2-ORF3 intron.}

F A Laski and G M Rubin

Genes Dev. 1989, 3:

Access the most recent version at doi:10.1101/gad.3.5.720

References This article cites 32 articles, 12 of which can be accessed free at: http://genesdev.cshlp.org/content/3/5/720.full.html\#ref-list-1

License

Email Alerting

Receive free email alerts when new articles cite this article - sign up in the box at the top Service right corner of the article or click here.

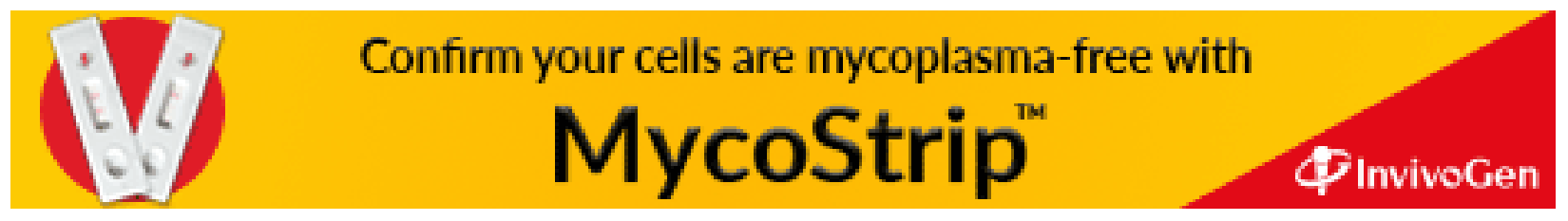

